\title{
Numerical Artifacts in Finite-Difference Time- Domain Algorithms Analyzed by Means of Principal Components
}

\author{
José Manuel López-Alonso, José María Rico-García, and Javier Alda
}

\begin{abstract}
Finite-difference time-domain (FDTD) algorithms are affected by numerical artifacts and noise. In order to obtain better results we propose the use of the principal component analysis based on multivariate statistical techniques. It allows a straightforward discrimination between the numerical noise and the actual electromagnetic field distributions, and the quantitative estimation of their respective contributions. Besides, the FDTD results can be filtered to clean the effect of the noise. The method has been applied successfully to two dimensional simulations: propagation of a pulse in vacuum using total field-scattered field techniques, and mode computation in a two-dimensional photonic crystal. In this last case, PCA has revealed hidden electromagnetic structures related to actual modes of the photonic crystal.
\end{abstract}

Index Terms-Filtering noise, finite-difference time-domain (FDTD) methods, photonic crystal, principal component analysis (PCA), total field-scattered field excitation.

\section{INTRODUCTION}

A S A DISCRETE approximation to Maxwell equations, the finite-difference time-domain (FDTD) method introduces numerical errors into the solutions: the fields computed step-bystep are affected by numerical artifacts, spurious high-frequency oscillations and transients induced by the modelization of field sources, interpolation errors, etc [1], [2]. Some of them arise from propagating wavelengths that remain unresolved in the grid, and their nature is linked to the spatial resolution of the computational grid; others have their origin in the finite duration of emitting sources, even adding undesirable frequency components to sinusoidal excitations. Managing such kind of artifacts and noise sources is a true challenge in the analysis of the results provided by FDTD. The methods to filter the effect of the noise are adapted to the electric properties and geometries of the materials. Therefore, a careful choice of the excitation source and a proper post-processing of the information achieved with the FDTD method are desirable. These methods can also be used to improve the quality of the results.

Manuscript received February 5, 2004; revised December 13, 2004. This work was supported in part by Project TIC 2001-1259 from the Ministerio de Ciencia y Tecnología of Spain.

J. M. López-Alonso is with the Optics Department, University Complutense of Madrid, School of Optics, 28037 Madrid, Spain (e-mail: jmlopez@opt.ucm.es).

J. M. Rico-García is with Optics Department of the University Complutense of Madrid, School of Optics, 28037 Madrid, Spain (e-mail: jmrico@ fis.ucm.es).

$\mathrm{J}$. Alda is with Optics Department of the University Complutense of Madrid, School of Optics, 28037 Madrid, Spain (e-mail: j.alda@ fis.ucm.es).

Digital Object Identifier 10.1109/TAP.2005.854555
The classification of the artifacts introduced by the numerical noise is an endless task. Their relevance depends on the point of view of the analysis and the purpose of the application. Then, instead of making an exhaustive classification, we will only describe a few typical sources of noise and numerical artifacts found frequently in FDTD computations.

In FDTD simulations the field source is necessary finite and discretized in the temporal domain. The finiteness of the temporal window broadens the spectrum of the source, even when it corresponds to a monochromatic stimulus. Some high-frequency components appear and propagate along the computational grid. In linear media, their contributions are independent and are added to the "real" electromagnetic fields. The discretization procedure may introduce sampling artifacts. Then, the spectrum will be aliased and distorted. So, the electric and magnetic fields contain unrealistic components generated by the method itself. Several tools have been proposed and used in order to "clean" this noise: smoothing windows [1], [3] for the source and application of digital filters to the fields resulting from the computation [4]. These methods reduce considerably the amount of the noise in the final results.

It is a commonplace in FDTD computations the use of the total field - scattered field zoning of the computational domain [5]. It provides memory savings in simulations and also allows a transparent source to be placed very close to the material structures. In many photonics applications, a pulsed or a continuous plane wave is injected to test the response of the device under study. This is done by means of an interpolating scheme over the limiting surface between the total field zone and the scattered field zone. This interpolation procedure generates noise around the limiting surface in the form of a spurious field. After some time steps, this noise contaminates the rest of the grid. This numerical error has been studied from the point of view of the theory of linear systems. It can be mitigated through a decimate filter or a similar approach [6].

In this paper, we describe a powerful method to deal with numerical artifacts and noise in FDTD calculations. It is based on a multivariate statistical technique called principal component analysis (PCA) [7]. The method has demonstrated to be very useful when characterizing noise in optical imaging systems [8]. PCA is not merely a filter technique. It is a method able to reveal variance structured ordered with respect to the contribution to the total variance of the data. It has been applied in social research, economics and where statistical analysis is needed. [9]-[11]. It provides results that can be easily interpreted in terms of images, temporal evolutions, and contri- 
butions to the total variance of the field. PCA method can be blindly applied to the output of the FDTD algorithms, without any a priori assumption. This is an advantage in the analysis because the method does not need adaptation to the practical situation under study. It identifies and classifies spatial-temporal structures that are present in any temporal sequence of spatial distributions of electromagnetic fields. PCA also quantifies the contribution of each spatial-temporal structure to the total variance of the original data set. An appropriate grouping of the results of the PCA method makes possible to distinguish those relevant contributions from the noise. Then, PCA becomes a tool to quantify the goodness of the FDTD simulation.

In Section II we have described the basic concepts of the PCA method that are needed in the analysis of FDTD results. Section III illustrates the application of the method for two cases. However, the method proposed here can be applied to any other situation. In the first case, the PCA method estimates quantitatively the numerical noise and associates it to spatial-temporal evolutions that can be filtered out. The second example deals with a photonic crystal. The results of the PCA have allowed the identification of hidden field structures linked to minimum asymmetries of the numerical model. These asymmetries are related with the use of an even or an odd number of points in rectangular computational grids. The PCA method has quantified the contribution of the asymmetric mode fields, and has identified their temporal frequencies. The results are in very good agreement with the published data obtained by other authors. Finally, Section IV summarizes the main conclusions of this paper.

\section{PRINCIPAL COMPONENT ANALYSIS}

The application of FDTD algorithms produces a sequence of frames $F\left(t_{i}\right)$ containing the values of the electromagnetic fields for a collection of time instants, $t_{i}$, where $i=1, \ldots, N$, being $N$ the total number of frames taken in the analysis. Each frame contains a number of sampled points that is given by the size of the spatial grid used in the FDTD algorithm. The $N$ frames treated by the PCA can be one-dimensional (1-D), two-dimensional (2-D), or three-dimensional (3-D), and in general they contain $M$ points. For the sake of simplicity, in this paper we will restrict our analysis to 2-D set of data having zero mean. This last condition does not mean a lack of generality because it is always possible to transform a nonzero mean data set into a zero-mean one by subtracting the mean value at each frame. This transformation can be reverted easily to retrieve the original or filtered data [8]. In general, due to the temporal evolution of the electromagnetic variables and its relation with the temporal period used to select the set of frames, these frames exhibit a non negligible covariance among them. Here is where PCA may help to better understand the results. Principal components are $N$ new uncorrelated variables obtained as linear combinations of the original ones [7]. Mathematically, the principal compo- nents $Y_{\alpha}$, also named as eigenframes, are linear combination of the original frames

$$
Y_{\alpha}=\sum_{t_{i}=1}^{N} e_{\alpha}\left(t_{i}\right) F\left(t_{i}\right)
$$

The coefficients of the linear combination $e_{\alpha}\left(t_{i}\right)$, are the eigenvectors obtained when solving the following:

$$
S e_{\alpha}=\lambda_{\alpha} e_{\alpha}
$$

where $\lambda_{\alpha}$ are the eigenvalues of the covariance matrix $\mathrm{S}$ calculated among frames [7], [8]. The elements of the covariance matrix are defined in the following way:

$$
S_{j k}=\left(\frac{1}{M-1}\right) \sum_{i=1}^{M}\left(f\left(t_{j}, r_{i}\right)-\bar{f}\left(t_{j}\right)\right)\left(f\left(t_{k}, r_{i}\right)-\bar{f}\left(t_{k}\right)\right)
$$

where $f\left(t_{j}, r_{k}\right)$ is the value of the signal at time $t_{j}$ in the point $r_{k} ; M$ is the number of points in the frame and

$$
\bar{f}\left(t_{j}\right)=\frac{1}{M} \sum_{i=1}^{M} f\left(t_{j}, r_{i}\right)
$$

is the mean of the frame.

The eigenvalues represent the variance of the corresponding principal component. Furthermore, in PCA, eigenvalues are obtained in a decreasing order. Then, the first one, $\lambda_{1}$, corresponds with the principal component that exhibit the largest variance. The relative contribution to the total variance explained by each principal component is defined as

$$
\Omega_{\alpha}=\frac{\lambda_{\alpha}}{\sum_{\alpha=1}^{N}} \lambda_{\alpha}
$$

One of the major advantages of PCA is that original frames can be reconstructed from the principal components, or eigenframes, by means of a process called rectification [8]

$$
F\left(t_{i}\right)=\sum_{\alpha=1}^{N} e_{\alpha}\left(t_{i}\right) Y_{\alpha}
$$

The sum in this equation can be restricted to a selected subset of the principal components. This selection allows the filtering of the original data.

Summarizing: the principal components, or eigenframes, are spatial distributions with the same units and within the same spatial domain than the original frames. The temporal evolution of a given eigenframe is described by the associated eigenvector, $e_{\alpha}(t)$. The amount of variance explained by the eigenframe is $\lambda_{\alpha}$.

Noise is assumed to be a contribution to the data that is independent from the signal, both temporally and spatially. Besides, the level of the noise is usually lower, or even much lower, than the level of the field components calculated in FDTD algorithms. Then, we expect that the first eigenvalues, eigenvectors, and eigenframes, are related with the actual electromag- 
netic fields, i.e., the signal. Accordingly, the noise should be associated with principal components different from the signal and labeled with greater indices. Equation (5) quantifies the relative contribution of the spatial-temporal structures associated with each principal component to the total variance of the set of frames.

A method to automatically group different components into processes is described in [8]. In that reference is explained that the eigenvalue uncertainty only depends on the number of realizations of the multivariate random variable (number of points in the computational grid). The number of frames determines the goodness of the sampling of the temporal evolution. This has been studied for the case of $1 / f$ noise [12], [13]. This grouping is based on the study of the uncertainties associated to each eigenvalue. If two principal components explain the same amount of variance, within their uncertainty, it could be possible to choose any other two related with the original one by a rotation with respect to a perpendicular axis. Then, it does not make any sense to reconstruct the frames by each one separately. Only the frames reconstructed by both principal components have a meaning. This is the key point of the grouping procedure. The uncertainties in the variance can be studied through the covariance matrix of the eigenvalues. This matrix is calculated through the actual realizations of the random variables (in this case the points of the computational grid) and the 4th order cumulants of them. Usually these cumulants are not very large and we may assume that the eigenvalues obtained for different realizations of the data follow a normal distribution. The mean of this normal distribution associated to each eigenvalue is $\mu=\lambda$, and the variance of the eigenvalue is given as $\sigma=5 \lambda^{2} / \mathrm{M}$, where is the number of realizations of the multidimensional variable (a detailed derivation of the eigenvalue grouping mechanism is presented in [8]). By this method, it is possible to define criteria for distinguishing consecutive eigenvalue. The proposed method is a generalization of the "scree test" [14]. This test selects the meaningful principal components by locating the eigenvalue where the consecutive differences between consecutive eigenvalues decrease abruptly. However, the scree test only gives qualitative criteria for grouping the principal components [14]. After applying the classification method into processes, the principal components are grouped together when the distance in variance between consecutive eigenvalues is less than their uncertainty. Besides, PCA method can filter the noise, and reconstructs the frames $F\left(t_{i}\right)$, without the principal components associated to the noise, by selecting in (4) the relevant eigenframes (this means to perform the linear combination with only the selected principal components).

In FDTD calculations, the knowledge about the material and geometrical structures, along with the spatial and temporal properties of the excitation, makes possible to identify the results with good judgment about its validity. This means that we can easily know if the obtained result makes good sense. This is an important issue in the applicability of the PCA method. PCA is a blind method that can be used without a prior knowledge of the practical situation. However, when analyzing the outputs of the PCA, the conditions of the simulation and the physical structures under study are necessary to properly understand the results. Besides, some kind of filtering can be used to clean-up the noise and preserve the useful information. PCA gives to this post-processing analysis the tools to identify those contributions having the largest variance, and therefore representing actual electromagnetic fields. Furthermore, PCA may reveal spatial-temporal structures that could be hidden in the set of data. The capability of the PCA to produce spatial distributions, the eigenframes, is strongly appreciated when analyzing the results. Besides, the spatial-temporal structures related with computational noise, or numeric artifacts are also identified and their contribution is estimated quantitatively, as we demonstrate in the following section.

\section{ApPlication of the Method}

In this section we apply the PCA to the identification and estimation of numerical noise and artifacts in FDTD algorithms. We have illustrated the PCA with two significant applications. In the first one, we have analyzed the numerical noise associated with the use of the total field-scattered field techniques. In the second case, we have analyzed the confined modes existing in a photonic crystal. We will check how the location of the excitation point with respect to the symmetry of the computational grid influences on the excited modes, and how the PCA is able to reveal field distributions that are hidden within the data.

These two examples illustrate two different ways of introducing the energy into the grid. In both of them, we will need an effective absorbing boundary condition that is implemented as a uniaxial perfect matched layer (UPML). The geometry of the grid for the calculation is a 2-D grid. The electromagnetic fields studied here correspond with TM modes, i.e., only $E_{z}, H_{x}$, and $H_{y}$ are non zero. In the following, we will focus our attention on the $E_{z}$ component.

\section{A. Total Field-Scattered Field}

In this case, the FDTD is applied on a 2-D square grid having $222 \times 222$ points with a spatial step of $\Delta x=\Delta y=0.025 \mu \mathrm{m}$, and Courant factor of 0.7068 . Specifically, the time domain signature of the source employed to inject energy into the grid is

$$
S(t)=\theta(t) \cos (2 \pi \nu t) e^{-\left(\frac{t}{T}\right)^{2}}
$$

where $\nu=1.07 \times 10^{14} \mathrm{~Hz}$ and $T=1.4735 \times 10^{-14}$ s. $\theta(t)$ denotes the Heaviside function. This function is included to make the signal causal, i.e., $S(t<0)=0$. The electric field has only omponent with an amplitude of $1 \mathrm{~V} / \mathrm{m}$. The pulse has a plane wavefront. The wave vector subtends an angle of $60^{\circ}$ with respect to the axis. The simulation time comprises 1000 temporal steps, and the analyzed data are 50 snapshots taken every 20 temporal steps.

Fig. 1 shows the \#15 frame of the simulation. A first analysis of the results obtained after applying the PCA method can be done from the eigenvalues. Fig. 2 presents the eigenvalues with their uncertainty values. In the left of the figure, they are shown in linear scale; in the right, in logarithmic scale. In the linear scale, it is shown clearly that the first four principal components explain the majority of the variance of the set: $92.44 \%$ of the total variance. These principal components are grouped in two different processes due to the fact that their variances are the 


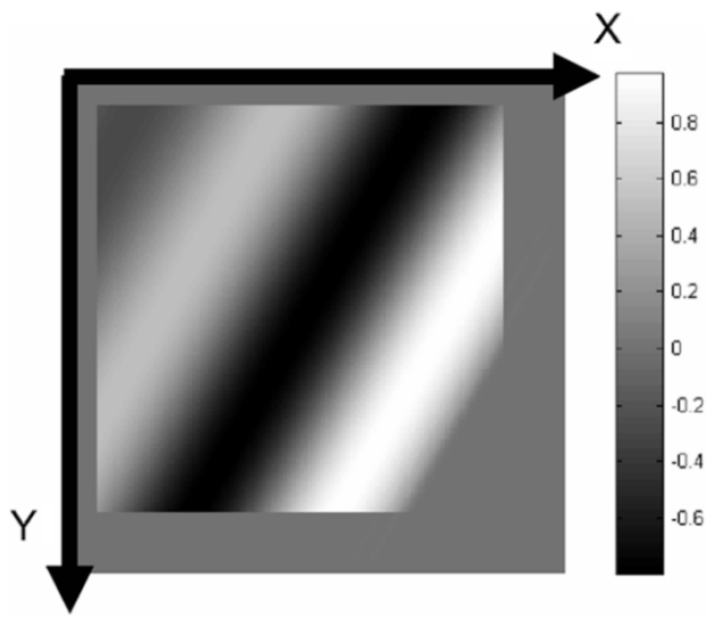

Fig. 1. Amplitude of the electric field, $E_{z}$, is represented as a greyscale map at the frame \#15. The $X$ and $Y$ directions are the same for all the electric field maps represented in this paper.

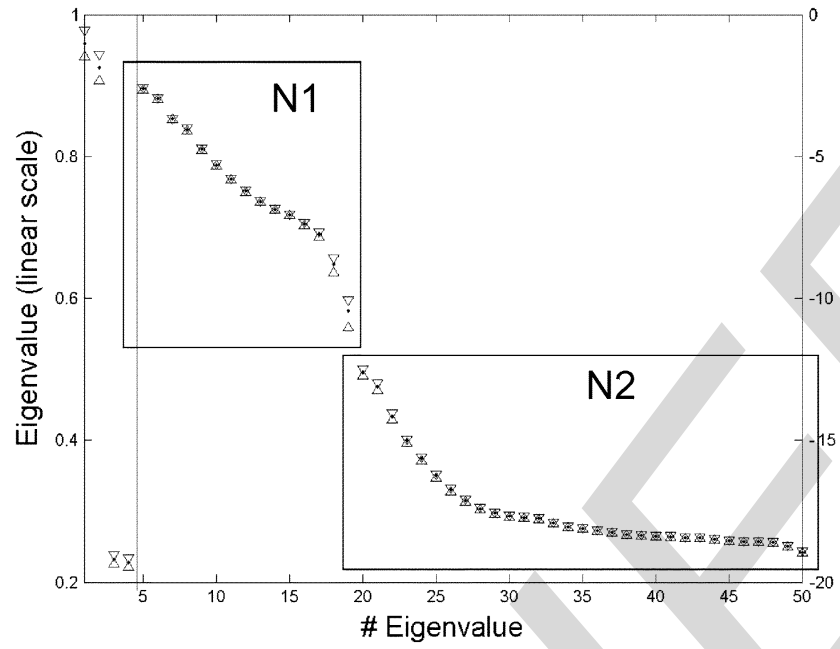

Fig. 2. Plot of the 50 eigenvalues obtained from the PCA method. The first four eigenvalues (at the left of the figure) are grouped in two processes by means of their uncertainty. The rest of the eigenvalues are represented in logarithmic scale and are grouped in two sets, $N 1$ and $N 2$, after analyzing the results.

same within the uncertainty [8]. The associated principal components are shown in Fig. 3. They represent the field that we are simulating: the spatial frequencies appearing in them are clearly related with the ones in the original image shown in Fig. 1.

The pairs of eigenvectors associated with them are shown in Fig. 4. They are the coefficients in the linear expansion of (6). These eigenvectors can be identified with the temporal evolution of the input Gaussian pulse introduced in the excitation. The presence of a similar, but shifted, temporal evolution explains the actual movement of the crossing pulse along the grid [7], [8].

The rest of the principal components can be related with some sort of computational noise. But even inside of this subset we can distinguish different spatial structures. The principal components $\# 5, \# 15, \# 30$, and $\# 50$ are shown in Fig. 5. It can be seen that principal components \#5 and \#15 still have a pulse-like shape, but their spatial frequencies are quite different from the original one (see Fig. 1). Moreover, they start to exhibit noise

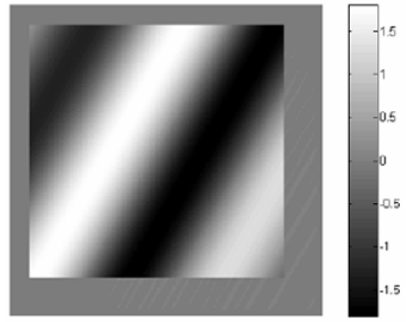

Y1

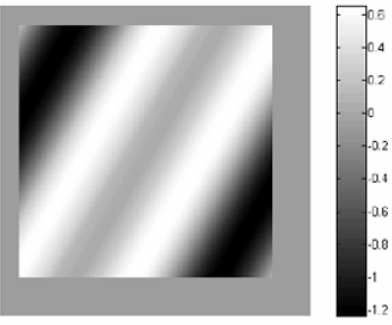

Y3

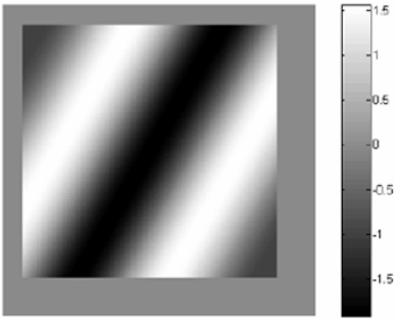

Y2

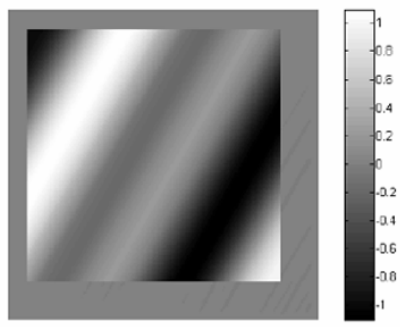

Y4
Fig. 3. First four eigenframes are given in thse maps. They are arranged together into two processes. They represent $92.44 \%$ of the total variance of the data.
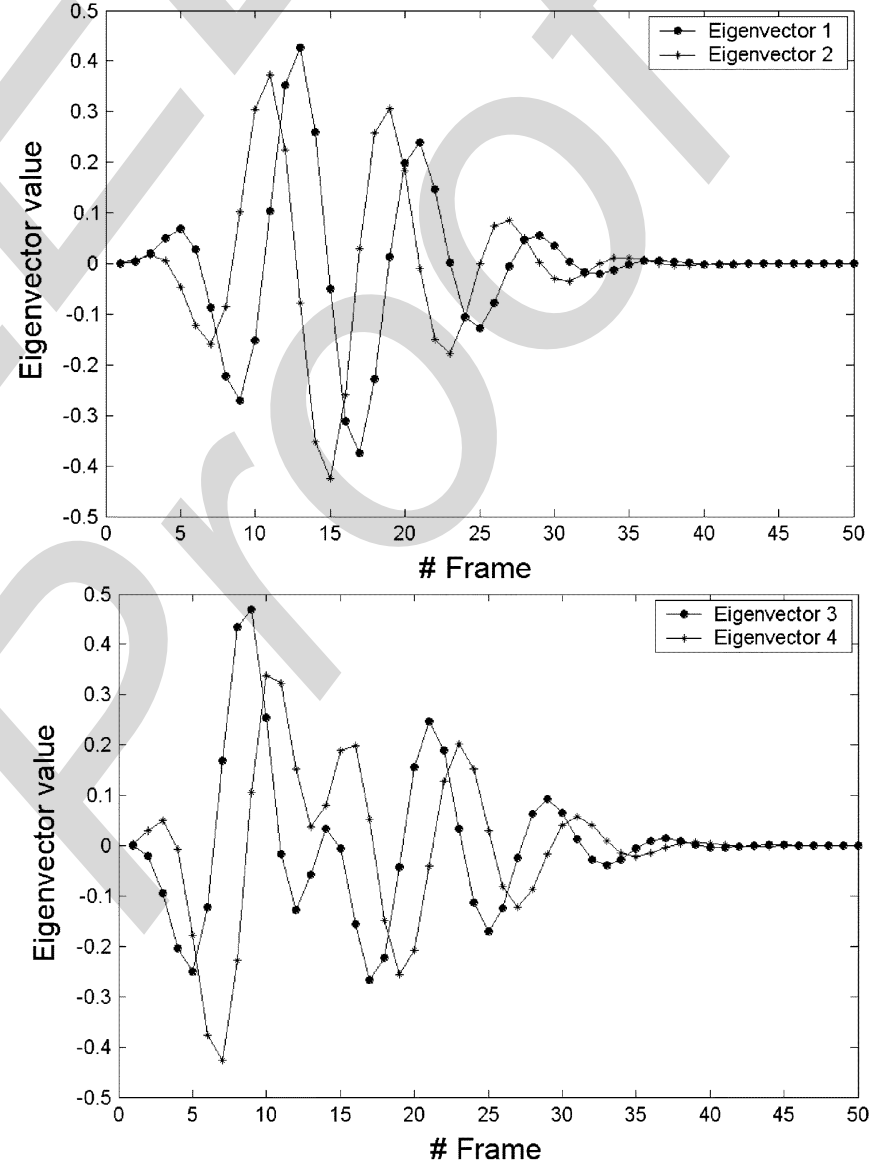

Fig. 4. Contribution of each one of the previous eigenframe to the original data at each time instant is given by the associated eigenvectors that are represented in this figure.

without a clear spatial structure. This noise also appears in the 

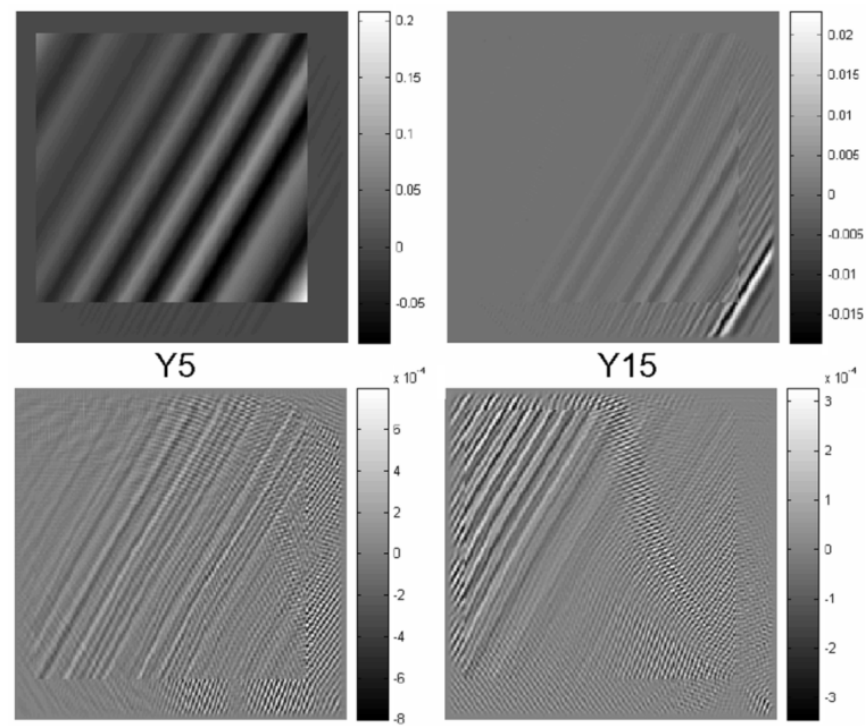

Y30

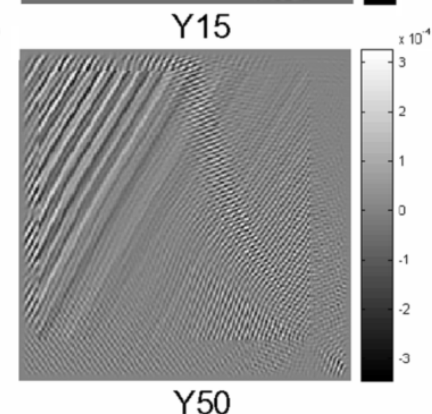

Fig. 5. Map of the electric field associated with the eigenframes \#5, \#15, \#30, and $\# 50$.

scattered-field zone, where the electric field should be zero because there are not any scatter elements inside the total-field zone. Principal components \#30 and \#50 can be clearly identified with noise, due to their spatial structure. Although the spatial structure is not random, these variations are of much higher spatial frequency than the excitation. Besides, its amplitude is of the same order than the amplitude of the granular structure of the noise. The eigenvalue of this principal component is negligible and due to the grouping algorithm, all this principal components are connected and cannot be considered individually. That is the reason why we have classified the computational noise into two categories. The first one is labeled as $N 1$, more related with the appearance of "spurious frequencies." The second one, named as $N 2$, is more related with the numerical noise due to the fact that their spatial structure follows the granularity of the grid used to simulate the problem. The first $N 1$ group represents the $7.56 \%$ of the total variance of the data, and the second one explains only $0.0003 \%$ of the total variance. This difference can also be understood by looking to the logarithm representation of the eigenvalues of Fig. 2.

Summarizing the results of this example, we have seen how the PCA method has discriminate the presence of the pulse and it has described the most of propagated electric field by using just four principal components. The other principal components have been identified as noise. Besides, the contribution of the different noise processes and types can be estimated. This fact supports a measurement of the accuracy of the method, and therefore a quantitative evaluation of the quality of the simulation. It also makes possible to extract very low level spatial-temporal structures.

\section{B. Photonic Crystal}

In this second case, the method is applied to test a different feature. The system under analysis is a photonic crystal with a central defect (see Fig. 6). It corresponds with a lossy microcavity structure. This type of inhomogeneous system has been

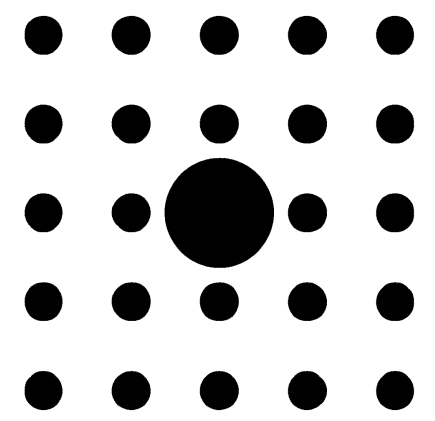

Fig. 6. Geometrical arrangement of the dielectric cylindrical structures that configure the photonic crystal. The period of the lattice is $1 \mu \mathrm{m}$. The excitation source is located at the center of this structure to excite the monopolar mode.

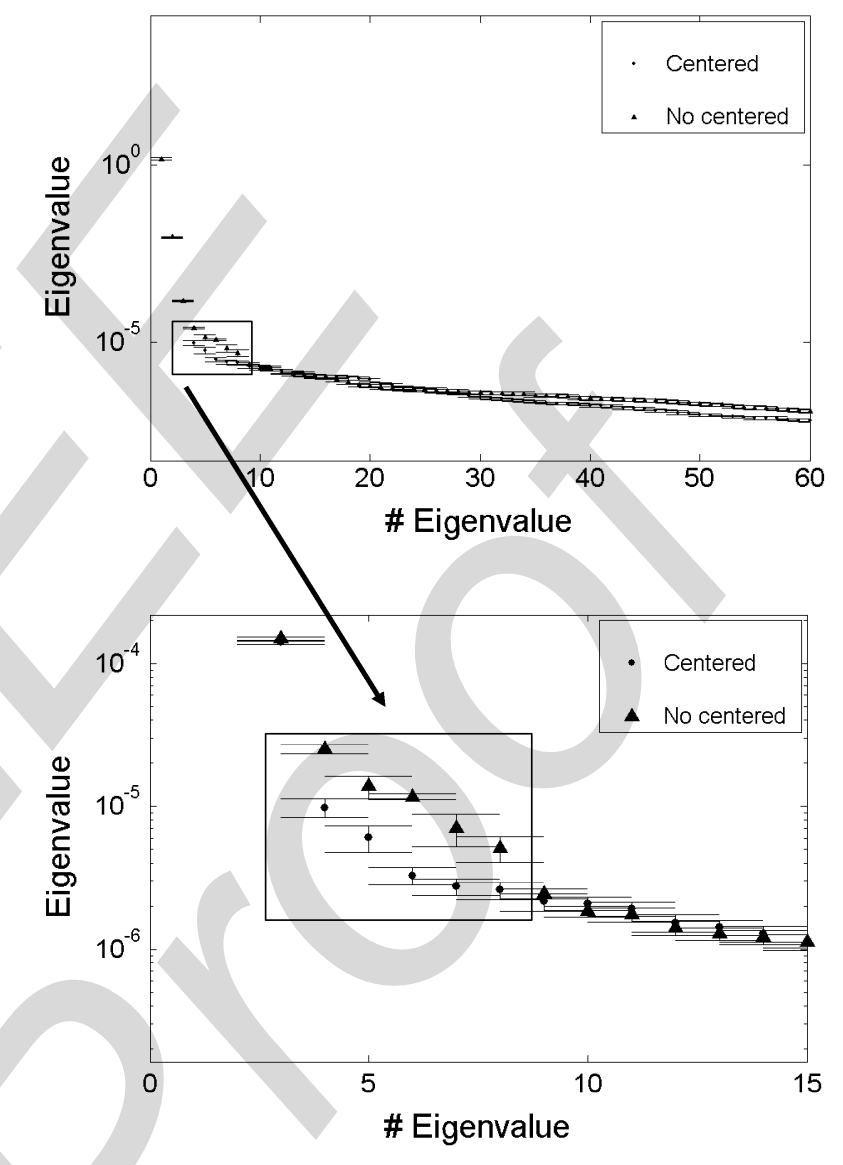

Fig. 7. Logarithmic plot of the first 60 eigenvalues obtained after applying the PCA method to the centered and decentered cases for a photonic crystal.

studied and analyzed in the literature by other methods [4]. The excitation is a soft source located at the center of the structure. The use of a soft source diminishes the effect of the spurious retro-reflections from the source. The grid for implementing the FDTD algorithm is a square grid. We have analyzed two cases, one with an even number of nodes along the grid, $222 \times 222$, and the other with an odd number, $221 \times 221$. The spatial step is the same for both cases, $\Delta x=\Delta Y=0.025 \mu \mathrm{m}$, and the Courant factor is 0.7068 . What we want to prove is the influence of the approximate centering of the source that can be obtained with an even number of nodes (decentered case), and the exact centering of the excitation source that can be obtained in the other case (centered case). The soft source is switched OFF after 5000 
TABLE I

RELATIVE IMPORTANCE OF THE EIGENVALUES OBTAINED FROM THE PCA

\begin{tabular}{ccc}
\hline \# PC & Centered (\%) & No centered (\%) \\
\hline 1 & 99.387 & 99.3533 \\
2 & 0.5993 & 0.6302 \\
3 & 0.0096 & 0.0101 \\
\hline 4 & 0.0007 & 0.0017 \\
\hline 5 & 0.0004 & 0.0009 \\
6 & 0.0002 & 0.0007 \\
7 & 0.0002 & 0.0005 \\
8 & 0.0002 & 0.0003 \\
\hline $9-101$ & 0.0017 & 0.0019 \\
\hline
\end{tabular}

time steps in order to prevent as much as possible the influence of spurious reflections [4], [5]. This limitation in the duration of the excitation broadens the spectrum. Therefore, the source is quasimonochromatic at a central frequency of $1.0035 \times 10^{14}$ $\mathrm{Hz}$, and having an amplitude of $1 \mathrm{~V} / \mathrm{m}$ for its electric field. The $E_{z}$ component is calculated and saved as a snapshot every 10 temporal steps. The simulations have been running until 41000 temporal steps and we have only taken the last portion between 40000 and 41000 [4]. We wait this time to avoid the transient regime and analyze an electric field distribution already stabilized within the microcavity.

In a photonic crystal, the symmetries of the lattice are related with the eigenmodes of the system. An eigenmode is a spatial field distribution whose evolution in time is quasiharmonic. The eigenmodes of our system have been calculated previously by other authors [4]. To test the influence of the even or odd grid in the eigenmode results we will apply the PCA method to the obtained electric field. The analysis of the eigenframes will reveal the influence of the centering of the source on the results.

The eigenvalues and their uncertatinties for both simulations are shown in Fig. 7. It is possible to see that there are three types of eigenvalues: 1) A first group that is the same for both simulations. They comprise principal components labeled \#1, $\# 2$, and \#3;2) A second group that differs from the centered to the decentered case. They comprise principal components from \#4 to \#8; and 3) The third group includes the most of the principal components into a single process. This last group is essentially related to the computational noise introduced in the system. Their associated images show the same grid granularity than those already presented in the previous case.

The relative amount of the total variance represented by these groups of principal components is shown in Table I. as a percentage. The values are given for both the centered and decentered cases.

From Fig. 7 and Table I it is possible to see that the first three principal components represent the same amount of variance, within the calculated uncertainty, in both simulations. Even more, after mapping the spatial distribution (principal components) of these three principal components they look similar (see Fig. 8). The first principal component appears associated to the excitation frequency and it has been found by other authors [4].

Principal components \#4-\#8 show the differences between the centered and decentered cases (see Figs. 9-11). The principal components are quite different in both simulations and the amount of variance explained by them differs in both sets too
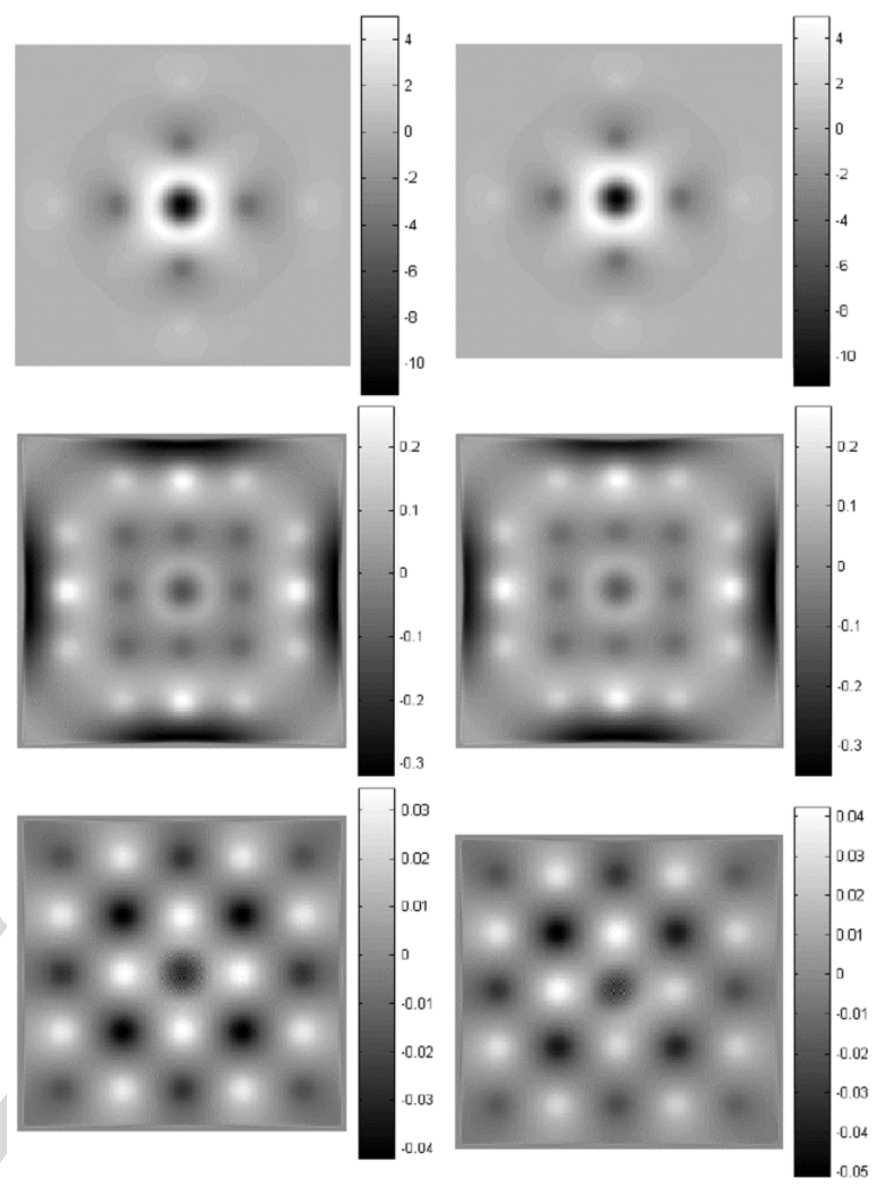

Fig. 8. Map of the $E_{z}$ component associated with the principal components \#1 (top), \#2 (middle), and \#3 (bottom). The left column corresponds with the centered case and the right column is for the decentered one.

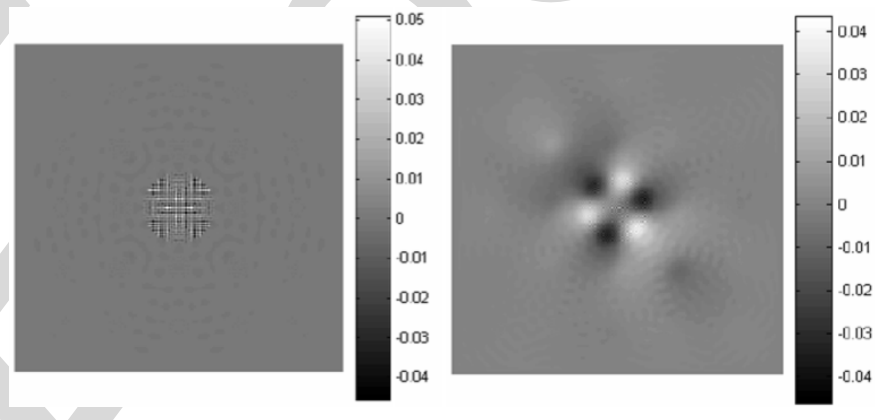

Fig. 9. Map of the $E_{z}$ component associated with the eigenframe \#4. The left column is for the centered case and the right column is for the decentered one.

(see Table I). For the decentered case, the application of the concept of process groups these principal components in three different processes. The eigenframe \#4 appears alone meanwhile $\# 5$ and \#6 are together and also \#7 and \#8 are within the same process. The principal component \#4 presents the most relevant feature (see Fig. 9). It resembles the hexapolar mode already referred in the literature and associated with an excitation that is not centered with respect to the symmetry of the photonic crystal [4]. According to this interpretation, it should be associated to a temporal frequency (see Fig. 12). Then, its eigenvector should be a quasiharmonic function. A ratio between the frequencies associated to the monopolar mode and the hexapolar mode can 


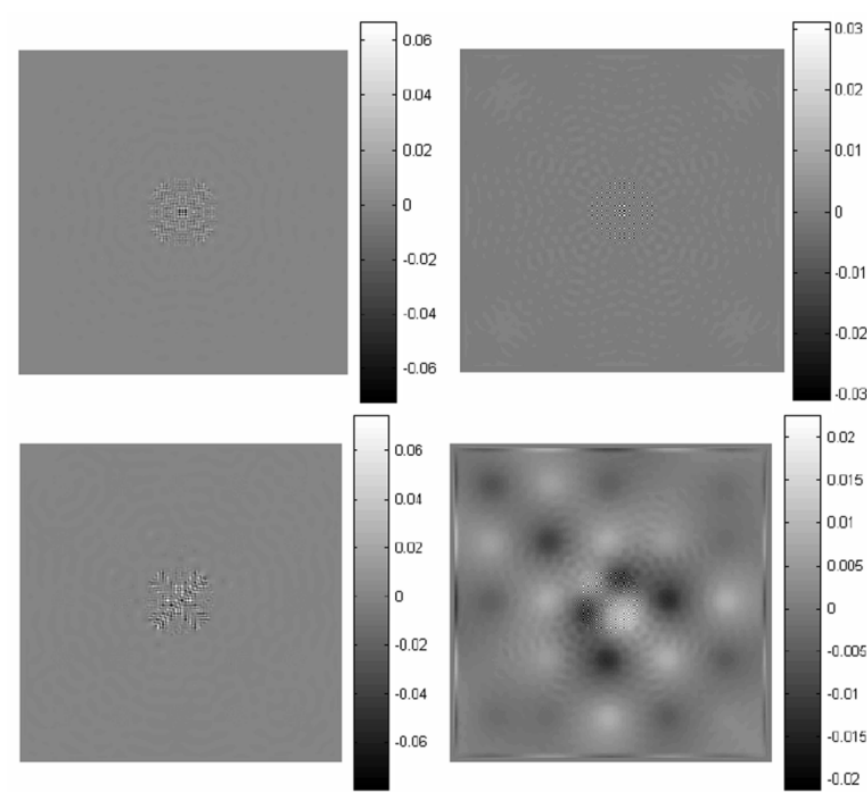

Fig. 10. Maps of the $E_{z}$ component for eigenframes \#5 (top) and \#6 (bottom). The left column is for the centered case and the right column is for the decentered one.

be calculated from the existing literature [4]. The referenced value of this ratio is 1.17. After applying the PCA method and comparing the frequencies associated with the eigenframe \#1 (identified with the central mode), and the eigenframe \#4 (identified with the hexapolar mode), it produces a ratio of 1.14 that is in very good agreement with the referenced results [4]. This effect can be seen as an actual excitation of the hexapolar mode due to the decentering of the grid by only one half of the spatial step. Moreover, the PCA method permits to evaluate the amount of uncertainty introduced by this choice in the calculation parameters. In this case, this mode represents the $0.0017 \%$ of the variance of the total data. Such a low level contribution would be filtered by a classic method of averaging. The interactions between this mode and the numerical noise in the grid are also present in eigenframe \#6 and disappear when we move to contributions of lower degree (see principal components shown in Figs. 10 and 11 for the decentered simulation).

\section{CONCLUSION}

FDTD algorithms are affected by intrinsic deviations due to the computational noise. In this paper we have applied the PCA method for analyzing the results provided by FDTD. The input for the PCA method is a sequence of frames, spaced regularly in time, representing a component of the electric or magnetic fields. The results of the PCA are interpreted in terms of the temporal and spatial properties of the electromagnetic fields. PCA method produces as many eigenvalues, eigenvectors, and eigenframes as the number of analyzed frames. In a previous contribution we defined a way to group the obtained set of eigenframes into processes. A process is a spatial-temporal structure that explains a given amount of variance of the original data. A process groups together different eigenframes when the associated eigenvalues overlap within their given uncertainties.

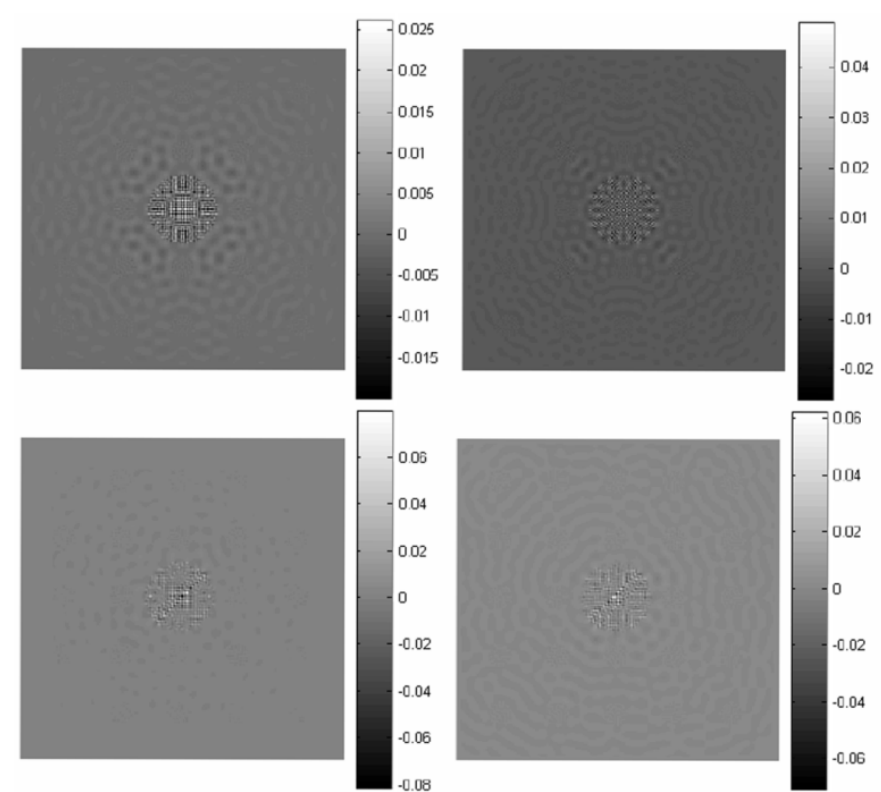

Fig. 11. Maps of the $E_{z}$ component for eigenframes \#7 (top) and \#8 (bottom) The left column is for the centered case and the right column is for the decentered one.

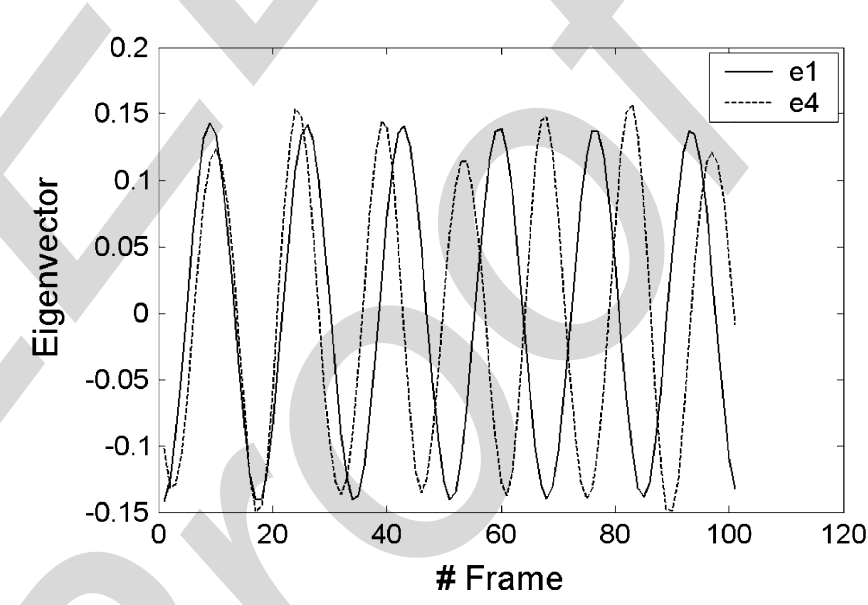

Fig. 12. Temporal contributions to the total frame set due to eigenframes $\# 1$ and \#4 are given by the associated eigenvectors. In this figure we have represented both of them shown their quasiharmonic dependence. This justifies the calculus of a temporal frequency associated to it.

Although the PCA method can be applied to any output of FDTD results, the demonstration of its usefulness for analyzing FDTD data has been illustrated with two significant examples. The first one studies the total field — scattered field technique that is widely used in FDTD. The PCA method has quantified the contribution of the numerical noise. It also provides a method to extract the actual pulse traveling through the computation grid and filter out some other noise contributions, with a quantitative evaluation of their importance. In the second example, a photonic crystal, the PCA has revealed the existence of hidden spatial-temporal structures. They could be excited even when a minimum lack of symmetry is present. The graphical output of the PCA method has made possible an easy and justified interpretation of the results. At the same time, the temporal 
evolution provided by the eigenvector has been linked to the actual time frequency of the analyzed example with a very good agreement.

The PCA method does not need any prior pre-processing of the FDTD data, nor any adaptation of the method itself. It is a blind procedure that can be applied to any temporal sequence of maps of a given electromagnetic field. The outputs of the PCA are analyzed taking into account the knowledge about the geometry and practical realization of the simulated structure and field excitation. This post-processing of the results makes possible to filter the data, to quantify the noise contribution, to identify noise sources and numerical artifacts, and to locate them both spatially and temporally. In this sense, we may say that PCA makes possible to evaluate the quality of FDTD simulations, showing a way to improve them.

Finally, we may conclude that PCA method not only provides a way to estimate quantitatively the artifacts induced by FDTD algorithms. It also gives spatial-temporal structures, different from the numerical artifacts (for example those presented in Figs. 3, 4, 8, and 12), that are expected to appear in actual experiments. The inputs to identify the physical origin of these structures can be found in the temporal evolution given by the eigenvectors and the spatial patterns given by the principal components. The detailed study of these findings is left to further research.

\section{REFERENCES}

[1] L. Gürel and U. Ogüz, "Signal-processing techniques to reduce the sinusoidal steady-state error in the FDTD method," IEEE Trans. Antennas Propag., vol. 48, no. 4, pp. 585-593, Apr. 2000.

[2] A. Taflove and K. Umashankar, "Radar cross section of general three-dimensional scatterers," IEEE Trans. Electromagn. Compat, vol. EMC-25, pp. 433-440, Apr. 1983.

[3] A. V. Oppenheim and A. W. Schafer, Discrete-Time Signal Processing. Englewood Cliffs, NJ: Prentice-Hall, 1989.

[4] S. Guo and S. Albin, "Numerical techniques for excitation and analysis of defect modes in photonic crystals," Opt. Express, vol. 11, pp. 1080-1089, May 2003.
[5] A. Taflove, Computational Electrodynamics: The Finite-Difference Time Domain Method, 2nd ed. Norwood, MA: Artech House, 2000.

[6] U. Ogüz, L. Gürel, and O. Arikan, "An efficient and accurate technique for the incident-wave excitations in the FDTD method," IEEE Trans. Microwave Theory Tech., vol. 46, no. 6, pp. 869-882, Jun. 1998.

[7] D. F. Morrison, Multivariate Statistical Methods, 3rd ed, Singapore: McGraw-Hill, 1990, ch. 8.

[8] J. M. López-Alonso, J. Alda, and E. Bernab́u, "Principal components characterization of noise for infrared images," Appl. Opt., vol. 41, pp. 320-331, Jan. 2002.

[9] E. R. Dougherty, Random Processes for Images and Signal Processing. Bellingham, WA: SPIE (Int. Soc. Opt. Eng.), 1998.

[10] L. A. Chan, N. M. Nasrabadi, and D. Torrieri, "Eigenspace transformation for automatic clutter rejection," Opt. Eng., vol. 40, pp. 564-573, 2001.

[11] R. Mitéran, J. P. Zimmer, F. Yang, and M. Paindavoine, "Access control: Adaptation and real-time implantation of a face, recognition method," Opt. Eng., vol. 40, pp. 586-593, 2001.

[12] J. M. López-Alonso and J. Alda, "Conditions for the applicability of the principal component analysis to the characterization of the $1 / f$," Noise, Fluctuation and Noise Letters, 2005, to be published.

[13] - "Operational parametrization of the $1 / f$ noise of a sequence of frames by means of the principal components analysis in focal plane arrays.," Opt. Eng., vol. 42, pp. 1915-1922, 2003.

[14] R. B. Cattell, "The scree test for the number of factors," J. Multivar. Behav. Res., vol. 1, pp. 245-276, 1966.

José Manuel López-alonso AUTHOR: PLEASE PROVIDE PLAIN TEXT BIOGRAPHIES OF THE AUTHORS AND EMAIL TO: d.menendez@ieee.org

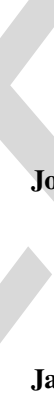

